\title{
The Pressure Signal Calibration TeChNology of THE Comprehensive Test SYSTEM
}

\author{
Yixiong $\mathrm{Xu}$
}

Mechatronic Engineering, Shanghai University of Engineering Science, Shanghai, China

\begin{abstract}
The pressure signal calibration technology of the comprehensive test system which involved pressure sensors was studied in this paper. The melioration of pressure signal calibration methods was elaborated. Compared with the calibration methods in the lab and after analyzing the relevant problems, the calibration technology online was achieved. The test datum and reasons of measuring error analyzed, the uncertainty evaluation was given and then this calibration method was proved to be feasible and accurate.
\end{abstract}

\section{KEYWORD}

Calibration online; Pressure transducer; Pressure signal calibration

\section{INTRODUCTION}

Integrated test system for pressure transducer can be achieved on liquid, gas, electric and other different media measurement and control of physical quantities, and it is mostly a complex testing system of electric hydraulic control. It are widely used in industrial control, military, aerospace and other fields. So the calibration of test system for pressure transmitter is particularly important. The improvement of calibration technology ensure system stability, accuracy and fast control of the parameters in a large extent, and provides quality assurance for research and production.

\section{The CALIBRATION OF PRESSURE SIgNALS OF THE INTEgRATED TEST}

\section{SYSTEM}

\subsection{Pressure signal improvement of the calibration method}

Calibration of the pressure signal is divided into two steps. The first step is a pressure transducer calibration; Calibration of pressure signal is the second one ${ }^{[1]}$. Conventional calibration method is DOI : $10.5121 /$ ieij.2016.4201 
to test the transducer that is removed from the system in the laboratory; the transducer is reinstalled in the test system after test qualified, and then through the pressure transducer, we calibrate the secondary display device of the test system. This calibration method is cumbersome, time consuming and have an accumulation of errors. All these lead to a large measurement error. Therefore we make the following improvement of the calibration method: Firstly, the pressure transducer is achieved on-line calibration instead of laboratory calibration; Secondly, the secondary display instrument of test systems is calibrated, by accessing directly to the terminal via a standard signal acquisition or signal source waveform generator.

\subsection{On-line calibration of pressure transmitter}

\subsubsection{Realization of environmental conditions}

Although pressure transducer calibration needs relatively harsh conditions, now many workshop works has requirements of environment temperature, humidity and outside magnetic field. Thus the calibration environment that temperature: $20^{\circ} \mathrm{C} \pm 5^{\circ} \mathrm{C}$, every $10 \mathrm{~min}$ change is not more than $1{ }^{\circ} \mathrm{C}$, relative humidity: $45 \%$ to $75 \%$, and there is no external magnetic field affecting the output stable mechanical vibration and no other effects in addition to the geomagnetic off-normal operation pressure transducer, can be achieved.

\subsubsection{Build on-line calibration device}

Calibration device consists of digital pressure calibrators, pressure source and composition of the adapter, and the auxiliary equipment is a walkie-talkie. CST 2005 digital pressure calibrator, intelligent modules and CST 1004 high pressure pump constitute the standard pressure source, which weighs $15 \mathrm{~kg}$ totally and is moved easily; Adapters is used to connected different thread diameters of standard pressure sensors to the source, and common diameter is $12 \mathrm{~mm}$ adapter turn $20 \mathrm{~mm}$; Sometimes the control system is relatively far away from the pressure transducer on site, and control system location apart more far, so two people are needed to complete the calibration. Calibration device from the system as shown in Figure 1.

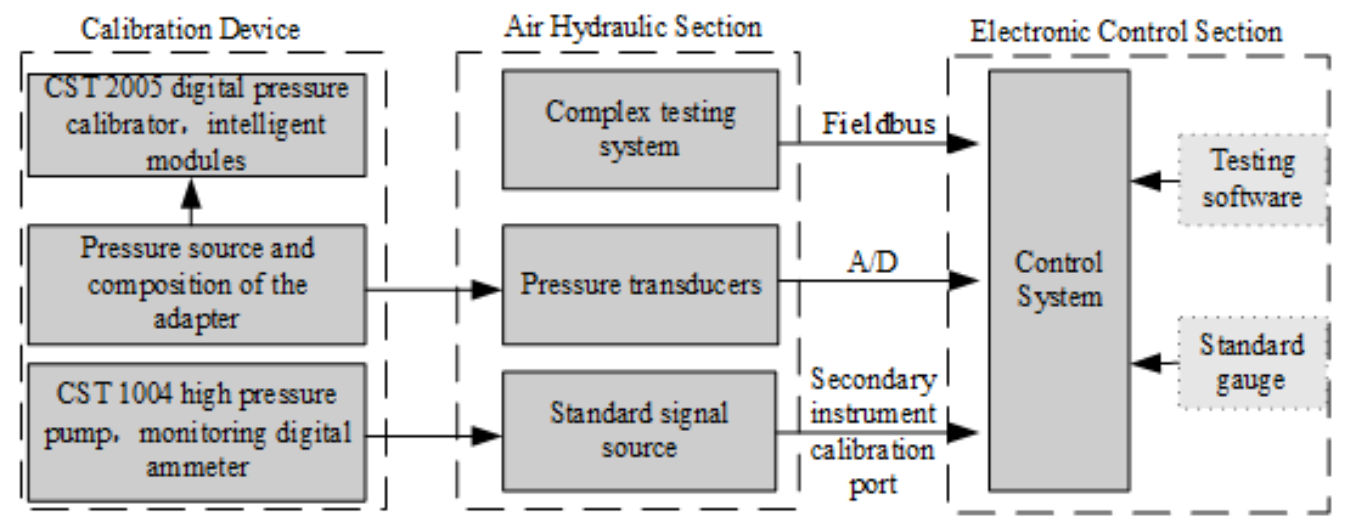

Fig.1 Composition of a test system and its calibration theory 


\subsubsection{The calibration method}

According to state test procedures, we finish the appearance and basic error checks. After calibration equipment and field equipment system get thermal equilibrium ( 2 hours), we installed and preheat the equipments, as shown in Figure 1.Because the pressure standard pressure source is difficult to maintain stable calibration points corresponding to the value in the measurement process, we use the method of multiple readings averaged to improve the accuracy of reading. Measurement error is calculated according to formula (1).If the test data meet the technical requirements, it is judged as qualified pressure transducer; Otherwise, it is considered unqualified.

$$
\triangle_{\mathrm{d}}=\mathrm{Y}_{\mathrm{d} 1}-\mathrm{Y}_{\mathrm{d} 2}
$$

In the formula: $\triangle_{\mathrm{d}}$-measurement error of the pressure transmitter calibrated point; $\mathrm{Y}_{\mathrm{d} 1}$ - the value of the standard digital pressure calibrator, $\mathrm{Pa} ; \mathrm{Y}_{\mathrm{d} 2}$ - pressure indication of the software interface or standard gauge display, $\mathrm{Pa}$.

\subsubsection{Advantages of pressure transducer calibration on-line}

It is very important to achieve pressure transmitter calibration on-line, which improves the efficiency of non-standard test equipment measurement ensures efficient conduct of scientific research and production. Compared to calibration in the laboratory, on-line calibration has the following advantages: firstly, through on-site demolition, on-site calibration and on-site installation, this method has shorten the calibration period and improved the calibration efficiency of the whole testing system; secondly, it is convenient to build the calibration device, not complicated; Thirdly, flexible, it is not influenced by other conditions apart from the calibration conditions.

\subsection{Current signal calibration}

The high-precision digital ammeter is connected in series circuit which is formed by standard signal source and pressure signal acquisition end of. Digital ammeter works as a data monitoring device and gets real-time data. Test software gives the current signal via the control panel; Record the measured value and displayed value and calculate the current linearity error. Since the electrical signal is small, in order to improve measurement accuracy and reduce human error machine error, in the calibration process we should pay attention to the following matters: Firstly, in the selection of monitoring digital ammeter, its resolution ratio should be more than four and a half, the resolution should be more than four and a half, its accuracy should be more than five ten thousandths, such as FLUKE8840;Secondly, when measuring we should try to avoid some of the factors that caused the difference, such as triboelectric effect, leakage current and piezoelectric effect. 


\section{Data Analysis}

\section{1 pressure transducer test data}

Taking 10Mpa as an example, test the effect of calibration techniques improved by actual test. Pressure transducer, measuring range: $0 \sim 10 \mathrm{Mpa}$, precision: $0.5 \%$.Test data in table 1.According to the technical requirements, The pressure transducer is determined as: Qualified.

Tab.1 test datum of 10Mpa pressure sensor

\begin{tabular}{|c|c|c|c|c|c|c|c|}
\hline $\begin{array}{c}\text { Serial } \\
\text { number }\end{array}$ & $\begin{array}{c}\text { Standard } \\
\text { pressure } \\
(\mathbf{M p a})\end{array}$ & Range & $\begin{array}{c}\text { First } \\
\text { time } \\
(\mathbf{M p a})\end{array}$ & $\begin{array}{c}\text { Second } \\
\text { time } \\
(\mathbf{M p a})\end{array}$ & $\begin{array}{c}\text { Third } \\
\text { time } \\
(\mathbf{M p a})\end{array}$ & $\begin{array}{c}\text { Mean } \\
\text { value } \\
(\mathbf{M p a})\end{array}$ & $\begin{array}{c}\text { Error } \\
(\%)\end{array}$ \\
\hline 1 & $0 \pm 0.05$ & Lower limit & 0.000 & 0.000 & 0.000 & 0.000 & 0 \\
\hline 2 & $1 \pm 0.05$ & $10 \%$ & 1.020 & 1.019 & 1.019 & 1.019 & -0.19 \\
\hline 3 & $2.5 \pm 0.05$ & $25 \%$ & 2.520 & 2.520 & 2.520 & 2.520 & -0.20 \\
\hline 4 & $5 \pm 0.05$ & $50 \%$ & 5.020 & 5.020 & 5.020 & 5.020 & -0.20 \\
\hline 5 & $7.5 \pm 0.05$ & $75 \%$ & 7.525 & 7.525 & 7.524 & 7.525 & -0.25 \\
\hline 6 & $9 \pm 0.05$ & $90 \%$ & 9.025 & 9.025 & 9.025 & 9.025 & -0.25 \\
\hline 7 & $9.5 \pm 0.05$ & Upper limit & 9.481 & 9.480 & 9.480 & 9.480 & 0.20 \\
\hline
\end{tabular}

\subsection{Current test data}

The test was made on the test data of the heat compressed air signal in the test station, whose acquisition range from 4 to $20 \mathrm{~mA}$. The data recorded in table 2 .

Tab.2 The test datum of current signal

\begin{tabular}{|c|c|c|c|}
\hline $\begin{array}{c}\text { Calibration } \\
\text { points }(\mathbf{m A})\end{array}$ & $\begin{array}{c}\text { Current signal Found } \\
(\mathbf{m A})\end{array}$ & $\begin{array}{c}\text { Current signal display } \\
(\mathbf{m A})\end{array}$ & Error (mA) \\
\hline 20 & 20.000 & 20.03 & 0.02 \\
\hline 16 & 16.000 & 16.01 & 00 \\
\hline 12 & 12.000 & 11.99 & -0.01 \\
\hline 8 & 8.000 & 7.98 & -0.02 \\
\hline 4 & 4.000 & 3.96 & -0.04 \\
\hline
\end{tabular}

\subsection{Evaluation of uncertainty in measurement}

\subsubsection{Sources of uncertainty}

a) The basic standard uncertainty: $u_{A}$;

b) The uncertainty of the introduction of the standard pressure source: $u_{b 1}$;

c) The measurement uncertainty introduced by the digital current meter: $\mathrm{u}_{\mathrm{b} 2}$; 


\subsubsection{Evaluation of uncertainty}

a) Basic standard type A evaluation of uncertainty

Since there are more test data, repeat the test for the pressure transducer 5Mpa profile and carry on the uncertainty analysis; The test data record as is shown in Table 3.

Tab. 4 repetitive test datum of 5Mpa of pressure sensor

\begin{tabular}{|c|c|c|c|c|c|c|c|c|c|c|}
\hline $\begin{array}{c}\text { Standard } \\
\text { pressure } \\
\text { (Mpa })\end{array}$ & 1 & 2 & 3 & 4 & 5 & 6 & 7 & 8 & 9 & 10 \\
\hline $\mathrm{X}_{\mathrm{i}}$ & 5.020 & 5.017 & 5.015 & 5.014 & 5.018 & 5.017 & 5.021 & 5.016 & 5.019 & 5.019 \\
\hline
\end{tabular}

Its average value:

$$
\bar{x}=\sum_{i=1}^{10} x_{i} / n=5.0176 \mathrm{Mpa}
$$

Standard type A evaluation of uncertainty calculated according to the formula(2):

$$
\begin{gathered}
s\left(x_{i}\right)=\sqrt{\sum_{i=1}^{n}\left(x_{i}-\bar{x}\right)^{2} /(n-1)} \\
s\left(x_{10}\right)=\sqrt{\sum_{i=1}^{10}\left(x_{i}-\bar{x}\right)^{2} /(10-1)}=\sqrt{0.00004548 / 9}=0.0022 \mathrm{Mpa}
\end{gathered}
$$

A class of uncertainty that measurement repeatability leads to is as follows:

$$
\mathrm{u}_{\mathrm{A}}=s\left(x_{10}\right) / \sqrt{n}=0.0007 \mathrm{Mpa}
$$

b) The uncertainty of the introduction of the standard pressure source: $\mathrm{u}_{\mathrm{b} 1}$

The main source is the intelligent pressure digital module of the standard pressure calibration instrument and the error of the digital pressure calibration instrument, while the influence of environmental temperature and humidity can be ignored. Type B evaluation of uncertainty. Standard pressure calibration instrument $15 \mathrm{Mpa}$ pressure module allows error limit $\mathrm{a}_{1}=0.003 \mathrm{Mpa}$. Its distribution is subject to uniform distribution, and take the factor $\mathrm{k}=\sqrt{3}$, calculated according to the formula(4):

$$
\mathrm{u}_{\mathrm{c} 1}=\mathrm{a}_{1} / \mathrm{k}=0.001732 \mathrm{Mpa}
$$

c) The measurement uncertainty introduced by the digital current meter:

Its main source is the uncertainty of the current introduction of the digital current meter in the measurement of the pressure signal acquisition port and the standard signal source, and its type B evaluation of uncertainty. The error limit of the digital meter $100 \mathrm{~mA}$ measurement range is $0.01 \%$, And $\mathrm{a}_{2}=0.01 \mathrm{~mA}$, take the coverage factor $\mathrm{k}=\sqrt{3}$, calculated according to the formula (5) : 


$$
\mathrm{A}_{2} / \mathrm{k}=0.00577 \mathrm{~mA}
$$

Converted into pressure by linear conversion:

$$
\mathrm{u}_{\mathrm{c} 2}=0.00540 \mathrm{Mpa}
$$

d) Combined standard uncertainty $U_{c}$

Above all are independent and unrelated, and the combined standard uncertainty $\mathrm{U}_{\mathrm{c}}$ is calculated according to the formula (7) :

$$
\mathrm{U}_{\mathrm{c}}=\sqrt{u_{A}^{2}+u_{b 1}^{2}+u_{b 2}^{2}} \quad=0.00571 \mathrm{Mpa}
$$

e) Evaluation of expanded uncertainty

All the sources of uncertainty are uniform distribution, and take $\mathrm{k}=2$, according to the formula (8):

$$
\mathrm{U}=2 * \mathrm{U}_{\mathrm{c}}=0.0114 \mathrm{Mpa}
$$

f) The measuring result of the 5Mpa file of the pressure transducer is:

$$
(5.0176 \pm 0.0114) \mathrm{Mpa} \text {. }
$$

\subsubsection{Methods for reducing uncertainty of measurement}

Through the analysis of the measurement uncertainty, we can see that the measurement uncertainty $\mathrm{u}$ introduced by the digital current meter is relatively larger, the uncertainty of $\mathrm{u}$ introduced by the standard pressure source is relatively moderate., and the basic standard uncertainty is relatively smaller. In order to improve the accuracy of measurement and reduce the uncertainty of measurement, we have the following improvements: firstly, choosing high precision digital ammeter can effectively reduce the measurement uncertainty introduced by the digital current meter and the uncertainty of the introduction of the standard pressure source, reducing system error; Thirdly, the A/D acquisition card could be modified to ensure consistency between data acquisition and actual values.

\section{SUMMARY}

The containing pressure transducer test system calibration techniques were studied, focusing on analyses and improvements of the system testing pressure signal calibration method, and through the test data processing and analysis, it proves the feasibility and superiority of this calibration method. It improves the calibration efficiency of pressure transmitter test system and the stability and reliability of the test system in the test products, and has accumulated some calibration experience for this type of test system. 


\section{REFERENCES}

[1] Shanghai Institute of Measurement and Testing technology. JJG 882-2004 pressure transducer calibration specification [s]. China measurement Publishing House, 2004

[2] China Metrology Association. Data processing and measurement professional practice [m]. Beijing: China's quality watchdog, 2013

[3] Sheng Hong Jiang, Feng Yi.Online Calibration of the Sensor [j]. Electronic Design Engineering, 2012,43 (1): 34-37.

[4] Tian Hui, Subaoming. The Measurement and Calibration of the Pressure Sensor System [j]. Electronic Component \& Device Applications, 2008 (09): 44-46

\section{Author}

Yixiong Xu(1985-),postgraduate in Shanghai University of Engineering Science(SUES), the main research interest now is Instrumentation measurement technology, address: Room 1105,Build ing NO.243,Lane 777,Dushi road, Minhang district, Shanghai(201109), fixed 021-34628108, mobile 13681897190,email:heroexu@126.com 